\title{
Treatment rationale and design of the PROLONG study: safety and efficacy of pembrolizumab as first-line therapy for elderly patients with non-small cell lung cancer
}

\author{
Takeshi Masuda ${ }^{1}$, Kazunori Fujitaka ${ }^{1}$, Nobuhisa Ishikawa ${ }^{2}$, Kikuo Nakano ${ }^{3}$, Masahiro Yamasaki ${ }^{4}$, \\ Souichi Kitaguchi ${ }^{5}$, Ken Masuda ${ }^{6}$, Kakuhiro Yamaguchi ${ }^{1}$, Shinjiro Sakamoto ${ }^{1}$, Yasushi Horimasu ${ }^{1}$, \\ Shigeo Kawase ${ }^{7}$, Shintaro Miyamoto ${ }^{1}$, Taku Nakashima ${ }^{1}$, Hiroshi Iwamoto ${ }^{1}$, Naoki Shiota ${ }^{8}$, \\ Tadashi Senoo ${ }^{9}$, Yoshikazu Awaya ${ }^{10}$, Tomohiro Kondo ${ }^{11}$, Takashi Yoshida ${ }^{12}$, Hironobu Hamada ${ }^{1}$, \\ Isao Murakami ${ }^{13}$, Noboru Hattori ${ }^{1}$
}

${ }^{1}$ Department of Respiratory Internal Medicine, Hiroshima University Hospital, 1-2-3 Kasumi, Minami-ku, Hiroshima, 734-8551, Japan; ${ }^{2}$ Department of Respiratory Medicine, Hiroshima Prefectural Hospital, 1-5-54 Ujinakanda, Minami-ku, Hiroshima, 734-8530, Japan; ${ }^{3}$ Department of Respiratory Internal Medicine, Kure Medical Center, 3-1 Aoyama, Kure, 737-0023, Japan; ${ }^{4}$ Department of Respiratory Internal Medicine, Hiroshima Red Cross Hospital \& Atomic-bomb Survivors Hospital, 1-9-6 Sendamachi Naka-ku, Hiroshima, 730-8619, Japan; ${ }^{5}$ Department of Medical Oncology, Hiroshima City Asa Citizens' Hospital, 2-1-1 Kabeminami, Asakita-ku, Hiroshima, 731-0293, Japan; ${ }^{6}$ Department of Respiratory Internal Medicine, Hiroshima City Hiroshima Citizens Hospital, 7-33 Motomachi, Naka-ku, Hiroshima, 730-8518, Japan; ${ }^{7}$ Department of Respiratory Internal Medicine, Kure Kyosai Hospital, 2-3-28 Nishichuo, Kure, 737-8505, Japan; ${ }^{8}$ Department of Respiratory Internal Medicine, Chugoku Rosai Hospital, 1-5-1 Hirotakaya, Kure, 737-0193, Japan; 'Department of Clinical Oncology, Hiroshima University Hospital, 1-2-3 Kasumi, Minami-ku, Hiroshima, 734-8551, Japan; ${ }^{10}$ Department of Respiratory Internal Medicine, Miyoshi Central Hospital, 10531 Higashisakaya, Miyoshi, 728-8502, Japan; ${ }^{11}$ Department of Respiratory Internal Medicine, JA Hiroshima General Hospital, 1-3-3 jigozen, Hatsukaichi, 738-8503, Japan; ${ }^{12}$ Department of Respiratory Internal Medicine, JA Onomichi General Hospital, 1-10-23, Hirahara, Onomichi, 722-8508, Japan; ${ }^{13}$ Department of Respiratory Internal Medicine, Higashihiroshima Medical Center, 513, Jike, Higashihiroshima, 739-0041, Japan

Contributions: (I) Conception and design: T Masuda, K Fujitaka, N Hattori; (II) Administrative support: None; (III) Provision of study materials or patients: All authors; (IV) Collection and assembly of data: None; (V) Data analysis and interpretation: T Masuda, K Fujitaka; (VI) Manuscript writing: All authors; (VII) Final approval of manuscript: All authors.

Correspondence to: Takeshi Masuda. Department of Respiratory Internal Medicine, Hiroshima University Hospital, 1-2-3 Kasumi, Minami-ku, Hiroshima 734-8551, Japan. Email: ta-masuda@hiroshima-u.ac.jp.

Background: Pembrolizumab is recommended as first-line therapy for patients with advanced non-small cell lung cancer (NSCLC) and a Programmed cell death ligand-1 (PD-L1) tumor proportion score (TPS) of $\geq 50 \%$ without driver mutations. However, the safety and efficacy were not investigated among patients who were $\geq 75$ years old.

Methods: This open-label single-arm phase II study is designed to evaluate pembrolizumab as first-line therapy for patients who are $\geq 75$ years old with advanced NSCLC and a PD-L1 TPS of $\geq 50 \%$ without driver mutations. The primary endpoint is progression-free survival, and the secondary endpoints are overall survival, objective response rate, safety, and quality of life. Recruitment started in October 2017 and is expected to continue for approximately 3 years.

Conclusions: Given the currently poor prognosis of elderly patients with advanced NSCLC, we hope that the findings of this study will facilitate more effective treatment in this setting.

Keywords: Pembrolizumab; elderly patient; non-small cell lung cancer (NSCLC)

Submitted May 23, 2019. Accepted for publication Oct 25, 2019.

doi: $10.21037 /$ jtd.2019.12.46

View this article at: http://dx.doi.org/10.21037/jtd.2019.12.46

(c) Journal of Thoracic Disease. All rights reserved. 


\section{Introduction}

Lung cancer is the most common cause of cancer-related death (1), and is classified into two broad histological types: non-small cell lung carcinoma (NSCLC) and small cell lung carcinoma. Patients with NSCLC, a performance status (PS) of $0-1$, and no driver mutations historically received a platinum-based doublet with third-generation chemotherapy as a standard treatment. However, an antibody to programmed cell death 1 (PD-1) and programmed cell death ligand 1 (PD-L1), which are immune checkpoint inhibitors, have been approved for treating advanced NSCLC. A randomized openlabel phase III trial (the KEYNOTE-24 study) compared pembrolizumab (the antibody to PD-1) and platinumbased doublet chemotherapy as first-line treatment for patients with advanced NSCLC, no driver mutations and a PD-L1 tumor proportion score (TPS) of $\geq 50 \%$ (2). The primary endpoint was progression-free survival (PFS), which was better in the pembrolizumab group than in the chemotherapy group (median: 10.3 vs. 6.0 months, hazard ratio: $0.50,95 \% \mathrm{CI}: 0.37-0.68 ; \mathrm{P}<0.001)$. In addition, several randomized phase III trials (the KEYNOTE-409, KEYNOTE-189, and IMpower-150 studies) compared platinum-doublet chemotherapy plus either PD-1/PDL1 antibody or placebo every 3 weeks for 4 or 6 cycles, followed by PD-1/PD-L1 antibody or placebo in NSCLC patients (3-5). These studies showed that the primary endpoint, PFS or overall survival (OS), was significantly longer in the PD-1/PD-L1 antibody-combination group than in the placebo-combination group, irrespective of PDL1 expression level. Thus, pembrolizumab or platinum doublet chemotherapy plus $\mathrm{PD}-1 / \mathrm{PD}-\mathrm{L} 1$ antibody is recommended as first-line treatment in this setting (6).

However, these studies did not consider patients who were $\geq 75$ years old. Lung cancer is primarily a disease of the elderly population, and the number of elderly patients aged 75 years or older with lung cancer is increasing (7). Previous in vitro and animal studies have shown that immune function weakens with increasing age $(8,9)$. Therefore, immunotherapy is considered to be less effective in elderly patients. Actually, a previous study involving squamous cell carcinoma patients treated with nivolumab after 1 st line treatment showed that median overall survival decreased in patients aged $>75$ years (5.8 months) compared to that in patients aged $<65$ years ( 8.6 months) $(10)$. However, contrastingly, the efficacy of nivolumab, as various lines of treatment, was not associated with age in lung cancer, renal cell carcinoma and melanoma patients (11-13). From these observations, there is uncertainty as to whether age is associated with the efficacy of immunotherapy. Therefore, we need to investigate the efficacy and safety of pembrolizumab as first-line therapy for patients aged $\geq 75$ years with advanced NSCLC.

A randomized phase 3 trial (the IFCT-0501 trial) including elderly patients with NSCLC reported that the platinumdoublet chemotherapy regimen improved survival, compared with the single-agent monotherapy regimen (14). However, the rate of toxic deaths was slightly higher in the doublet chemotherapy group than in the monotherapy group; the overall rate of hematological toxicities was also higher in the doublet chemotherapy group. Therefore, the National Comprehensive Cancer Network (NCCN) guidelines suggest that systemic chemotherapy for elderly patients needs to be selected carefully to avoid adverse reactions (6). Furthermore, in studies that compared platinum-doublet chemotherapy plus either PD-1/PD-L1 antibody or placebo administration, grade 3 or higher adverse events have been reported to be more frequent in the PD-1/PD-L1 antibody-combination group than in the placebo group. On the other hand, the KEYNOTE-024 study revealed that pembrolizumab was associated with fewer severe adverse events, relative to platinum doublet chemotherapy (26.6\% vs. $53.3 \%)$. These results suggest that pembrolizumab monotherapy may be safe for elderly patients with NSCLC.

\section{AIM}

We aimed to examine the efficacy and safety of pembrolizumab monotherapy for patients who are $\geq 75$ years old with untreated advanced NSCLC and a PDL1 TPS of $\geq 50 \%$ in this study. Unless the patient has received pembrolizumab, single-drug third-generation chemotherapy is recommended for patients who are $\geq 75$ years old with advanced chemotherapy-naïve NSCLC and no driver mutations, although this treatment was only associated with a PFS of approximately 4.3 months in the MILES and WJTOG 9904 studies $(15,16)$. Therefore, it is urgently needed to investigate whether pembrolizumab is effective and safety for elderly patients.

\section{Methods}

\section{Study design}

This open-label single-arm phase II study will evaluate the 


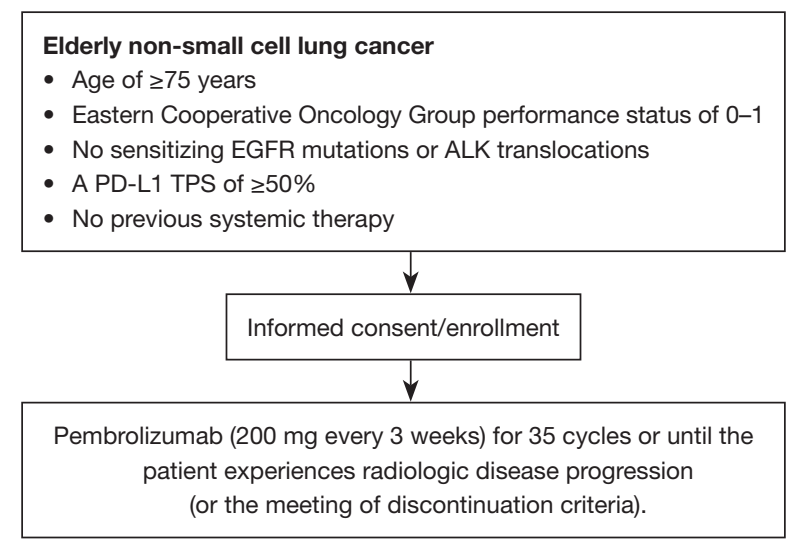

Figure 1 Study flow chart. EGFR, epidermal growth factor receptor mutations; ALK, anaplastic lymphoma kinase; PD-L1, programmed cell death ligand-1; TPS, tumor proportion score.

safety and efficacy of pembrolizumab as first-line therapy for Japanese patients who are $\geq 75$ years old with advanced NSCLC and a PD-L1 TPS of $\geq 50 \%$ without driver mutations. Figure 1 shows a flow chart of the study. Twelve hospitals agreed to take part in this study, and the study protocol was approved by the institutional review board of each hospital. We plan to recruit 26 patients between October 2017 and October 2020. The observational period is 1 year from the time of the final registration.

\section{Clinical measures}

We will collect:

(I) Baseline demographic and clinical information, including smoking status.

(II) Personal details and demographics including height, weight, and gender.

(III) Date of diagnosis.

(IV) NSCLC clinical stage, including the subtypes of NSCLC, tumor site, and number of metastases.

(V) Confirmation of eligibility.

(VI) Confirmation of written informed consent.

(VII) Comorbidity, ECOG performance status.

\section{Treatment}

Patients will receive intravenous pembrolizumab (200 mg every 3 weeks) for 35 cycles or until the patient experiences radiologic disease progression, experiences treatmentrelated adverse events of unacceptable severity, withdraws their consent, or treatment is terminated at the discretion of the investigator.

\section{Patient population, key eligibility criteria}

Inclusion criteria:

(I) Age of $\geq 75$ years at the time of informed consent.

(II) Eastern Cooperative Oncology Group performance status of $0-1$.

(III) Histologically confirmed stage IV or recurrent NSCLC.

(IV) No sensitizing epidermal growth factor receptor mutations or anaplastic lymphoma kinase translocations (analysis of $B R A F$ gene mutation or ROS-1 gene arrangement is not mandatory. However, we will collect data of the status of these genes).

(V) A PD-L1 TPS of $\geq 50 \%$ (22C3 pharmDx assay).

(VI) No previous systemic therapy.

(VII) Estimated life expectancy of $>3$ months.

(VIII) Adequate organ function.

(IX) Provision of written informed consent.

Exclusion criteria:

(I) Uncontrollable pleural effusion, ascites, or pericardial fluid.

(II) Multiple cancers.

(III) Untreated central nervous system metastases and/ or carcinomatous meningitis.

(IV) Evidence of severe or uncontrolled systemic disease.

(V) Evidence of interstitial pneumonia during chest computed tomography (CT).

(VI) Any major surgical procedure (as defined by the investigators) within 28 days of the first pembrolizumab dose. 
(VII) Underwent radiotherapy within 4 weeks before the first pembrolizumab dose

(VIII) Active autoimmune disease that has required systemic treatment using corticosteroids or other immunosuppressive medications.

\section{Patient registration}

After the patient's eligibility has been confirmed and informed consent has been obtained, the patient will be registered and begin receiving treatment. Patient recruitment began in October 2017 and is expected to continue for approximately 3 years.

\section{Evaluation of response and safety}

Patients must undergo various pre-treatment evaluations: a CT or magnetic resonance imaging scan of the brain, CT scans of the chest and abdomen, a bone scan or positron emission tomography scan, and electrocardiography. Patients will undergo tumor assessments at baseline and then every 3 cycles. The tumor response and/or radiologic disease progression will be evaluated based on version 1.1 of the Response Evaluation Criteria in Solid Tumors (17). Adverse events will be recorded using version 4.0 of the National Cancer Institute's Common Terminology Criteria for Adverse Events (18).

Quality of life will be assessed using EORTC QLQ-C30 and EORTC QLQ-LC13, with patients completing the questionnaires immediately after providing informed consent and then again before the third cycle, the sixth cycle, and week 18 .

\section{Statistical design}

Primary endpoint is PFS. The Kaplan-Meier method will be used to analyze PFS, which is defined as the interval from the first day of therapy to the first instance of treatment failure, which includes, death and disease progression.

Secondary endpoints were overall survival, overall response rate, disease control rate, safety and QOL. The statistical methods are following;

Overall response rate: the response rate (CR + PR ratio) is calculated, and the $95 \%$ confidence interval is also calculated.

Disease control rate: the disease control rate $(\mathrm{CR}+\mathrm{PR}+$ SD ratio) is calculated, and the $95 \%$ confidence interval is also calculated.

Safety: the adverse events observed during protocol treatment are summarized by type and grade.

Overall survival: the cumulative survival rate is calculated using the Kaplan-Meier method and we will calculate the 95\% confidence interval.

QOL: changes in QOL is analyzed for PPS with reference to pre-treatment.

\section{Sample size}

The sample size was determined based on an expected median PFS of 4.3 months for previous single-drug treatments $(15,16)$, an expected median PFS of 10.3 months in the present study, a two-sided alpha value of 0.05 , and power of 0.8. Based on these parameters, 24 patients will need to be enrolled within the first 3 years, with an additional 1 year of follow-up. Allowing for dropouts, 26 subjects will be enrolled.

\section{Ethics \& dissemination}

\section{Ethics and informed consent}

The trial received ethical approval from the institutional review board of Hiroshima University Hospital, Hiroshima, Japan (number C-207-2, the last edition ver $2.122 /$ Mar/2018) and was approved by the ethics committee of each participating institution. In addition, this study protocol conforms to the ethical principles outlined in the Declaration of Helsinki and has been registered in the University Hospital Medical Information Network Clinical Trials Registry database (UMIN0000029602). All patients will provide written informed consent before enrollment.

\section{Trial monitoring and oversight}

Monitoring will be conducted in accordance with "Standard Procedure for Monitoring in Clinical Research" and "Clinical Research Monitoring Implementation Manual" owned by the Hiroshima University Hospital General Medical Research Promotion Center. Once a year, the status of obtaining consent and the occurrence of adverse events will be submitted to the principle investigator and the administrator of the facility to which the principle investigator belongs.

\section{Dissemination}

The subgroup analysis of KEYNOTE-024 study showed that the progression-free survival benefit of the pembrolizumab in the patients who are $\geq 65$ years old. In addition, this study included patients over the age of 75 , the 
oldest was 90 (2). However, to the best of our knowledge, the present study is the first clinical trial to investigate the safety and efficacy of pembrolizumab as first-line therapy for patients who are $\geq 75$ years old with advanced NSCLC and a PD-L1 TPS of $\geq 50 \%$. We hope that the findings will facilitate more effective treatment in this setting. In addition, this study will identify the frequent and severe immune-related adverse events in this population.

\section{Study status}

This study was opened to recruitment in October 2017. As of April 2019, 15 subjects have been enrolled.

\section{Acknowledgments}

The authors thank the patients, their families, and the participating investigators. In addition, we thank Akiko Miyake (Department of Molecular and Internal Medicine, Graduate School of Biomedical \& Health Sciences, Hiroshima University, Hiroshima 734-8551, Japan) for support in this study.

Funding: None.

\section{Footnote}

Conflicts of Interest: The authors have no conflicts of interest to declare.

Ethical Statement: The authors are accountable for all aspects of the work in ensuring that questions related to the accuracy or integrity of any part of the work are appropriately investigated and resolved. The trial received ethical approval from the institutional review board of Hiroshima University Hospital, Hiroshima, Japan (number C-207-2, the last edition ver 2.122/Mar/2018) and was approved by the ethics committee of each participating institution. In addition, this study protocol conforms to the ethical principles outlined in the Declaration of Helsinki and has been registered in the University Hospital Medical Information Network Clinical Trials Registry database (UMIN0000029602). All patients will provide written informed consent before enrollment.

Open Access Statement: This is an Open Access article distributed in accordance with the Creative Commons Attribution-NonCommercial-NoDerivs 4.0 International License (CC BY-NC-ND 4.0), which permits the non- commercial replication and distribution of the article with the strict proviso that no changes or edits are made and the original work is properly cited (including links to both the formal publication through the relevant DOI and the license). See: https://creativecommons.org/licenses/by-nc-nd/4.0/.

\section{References}

1. Siegel RL, Miller KD, Jemal A. Cancer statistics, 2016. CA Cancer J Clin 2016;66:7-30.

2. Reck M, Rodriguez-Abreu D, Robinson AG, et al. Pembrolizumab versus Chemotherapy for PD-L1Positive Non-Small-Cell Lung Cancer. N Engl J Med 2016;375:1823-33.

3. Gandhi L, Rodriguez-Abreu D, Gadgeel S, et al. Pembrolizumab plus Chemotherapy in Metastatic NonSmall-Cell Lung Cancer. N Engl J Med 2018;378:2078-92.

4. Paz-Ares L, Luft A, Vicente D, et al. Pembrolizumab plus Chemotherapy for Squamous Non-Small-Cell Lung Cancer. N Engl J Med 2018;379:2040-51.

5. Socinski MA, Jotte RM, Cappuzzo F, et al. Atezolizumab for First-Line Treatment of Metastatic Nonsquamous NSCLC. N Engl J Med 2018;378:2288-301.

6. Netwook NCC. NCCN clinical practice guideline in oncology (NCCN guidelines ${ }^{\circledR}$ ) Non-small cell lung cancer. Version 2. 2018, December 192017 Available online: https://www.nccn.org/professionals/physician_gls/pdf/ nscl.pdf. Accessed February, 212018.

7. Novello S, Barlesi F, Califano R, et al. Metastatic nonsmall-cell lung cancer: ESMO Clinical Practice Guidelines for diagnosis, treatment and follow-up. Ann Oncol 2016;27:v1-27.

8. Lustgarten J. Cancer, aging and immunotherapy: lessons learned from animal models. Cancer Immunol Immunother 2009;58:1979-89.

9. Pawelec G, Lustgarten J, Ruby C, et al. Impact of aging on cancer immunity and immunotherapy. Cancer Immunol Immunother 2009;58:1907-8.

10. Grossi F, Crino L, Logroscino A, et al. Use of nivolumab in elderly patients with advanced squamous non-small-cell lung cancer: results from the Italian cohort of an expanded access programme. Eur J Cancer 2018;100:126-34.

11. Ibrahim T, Mateus C, Baz M, et al. Older melanoma patients aged 75 and above retain responsiveness to anti-PD1 therapy: results of a retrospective singleinstitution cohort study. Cancer Immunol Immunother 2018;67:1571-8.

12. Marur S, Singh H, Mishra-Kalyani P, et al. FDA analyses 
of survival in older adults with metastatic non-small cell lung cancer in controlled trials of PD-1/PD-L1 blocking antibodies. Semin Oncol 2018;45:220-5.

13. Vitale MG, Scagliarini S, Galli L, et al. Efficacy and safety data in elderly patients with metastatic renal cell carcinoma included in the nivolumab Expanded Access Program (EAP) in Italy. PLoS One 2018;13:e0199642.

14. Quoix E, Zalcman G, Oster JP, et al. Carboplatin and weekly paclitaxel doublet chemotherapy compared with monotherapy in elderly patients with advanced non-smallcell lung cancer: IFCT-0501 randomised, phase 3 trial. Lancet 2011;378:1079-88.

15. Gridelli C, Perrone F, Gallo C, et al. Chemotherapy for elderly patients with advanced non-small-cell lung cancer:

Cite this article as: Masuda T, Fujitaka K, Ishikawa N, Nakano K, Yamasaki M, Kitaguchi S, Masuda K, Yamaguchi K, Sakamoto S, Horimasu Y, Kawase S, Miyamoto S, Nakashima T, Iwamoto H, Shiota N, Senoo T, Awaya Y, Kondo T, Yoshida T, Hamada H, Murakami I, Hattori N. Treatment rationale and design of the PROLONG study: safety and efficacy of pembrolizumab as first-line therapy for elderly patients with non-small cell lung cancer. J Thorac Dis 2020;12(3):1079-1084. doi: $10.21037 /$ jtd.2019.12.46 the Multicenter Italian Lung Cancer in the Elderly Study (MILES) phase III randomized trial. J Natl Cancer Inst 2003;95:362-72.

16. Kudoh S, Takeda K, Nakagawa K, et al. Phase III study of docetaxel compared with vinorelbine in elderly patients with advanced non-small-cell lung cancer: results of the West Japan Thoracic Oncology Group Trial (WJTOG 9904). J Clin Oncol 2006;24:3657-63.

17. Eisenhauer EA, Therasse P, Bogaerts J, et al. New response evaluation criteria in solid tumours: revised RECIST guideline (version 1.1). Eur J Cancer 2009;45:228-47.

18. Common terminology criteria for adverse events: (CTCAE). v4.03. ed. [Bethesda, Md.]: U.S. Department of Health and Human Services; 2010. 